\title{
Non-contact 3D acquisition system based on stereo vision and laser triangulation
}

\author{
João L. Vilaça · Jaime C. Fonseca • \\ António M. Pinho
}

Received: 30 November 2007 / Accepted: 28 August 2008

(c) Springer-Verlag 2008

\begin{abstract}
This paper proposes a novel contact 3D acquisition system based on stereo vision and laser triangulation. The system is composed by several software modules for data acquisition purposes, data calibration, data processing and data reconstruction of 3D scenes. Different 3D image techniques, such as, polynomial determination, cubic spline interpolation and hierarchical space decomposition were used. To validate this method, a simple laboratory prototype machine was built for the purpose of road profile acquisition, road macro and mega texture characterization. In this paper, only the results and discussion of road profile acquisition are presented.
\end{abstract}

Keywords Calibration - Laser line detection .

$3 \mathrm{D}$ reconstruction $\cdot 3 \mathrm{D}$ measurements

\section{Introduction}

Nowadays, reverse engineering of physical objects has two principal and distinct goals: the comparison between real dimensions of an object and its model; the generation of computer-based models (CAD) from a physical object with complex geometry [12].

Some of these measurement techniques are based on surface scanning, which is carried out by a direct controlled

J. L. Vilaça $(\varangle)$ · J. C. Fonseca

Industrial Electronics Department, Minho University,

Azurem Campus, 4800-058 Guimarães, Portugal

e-mail: joaovilaca@dei.uminho.pt

A. M. Pinho

Mechanical Engineering Department, Minho University,

Azurem Campus, 4800-058 Guimarães, Portugal energy projection on the surface, using one or more projectors, and at least one sensor. Usually, these techniques require complicated calibration procedures for the evaluation of the parameters of the optical devices (sensors and light source) necessary for the measurement process [1].

Camera (sensor) calibration is understood as the process of determining the internal camera geometric and optical characteristics (intrinsic parameters) and/or the $3 \mathrm{D}$ position and orientation of the camera frame relative to a certain world coordinate system (extrinsic parameters). The purpose of the calibration is to set a relationship between $3 \mathrm{D}$ points from the world and $2 \mathrm{D}$ points from the image, seen by the computer $[5,6,10,11,13]$.

In 3D measurement systems, composed by one laser light source and one camera, the common procedure is to use the triangulation method (active vision technique) to establish the relationship between $2 \mathrm{D}$ points from the image and the $3 \mathrm{D}$ points from the world [4]. One of the major limitations of this technique is the occlusion problem, which occurs when the camera is not capable of visualizing the laser beam reflected by the object, due to the object geometry. To overcome this limitation, it is common to use a second camera. However, the use of two cameras involves a stereo match between points on the right image and points on the left image, associated with the same physical point of the scene 3D-the stereo matching problem.

The matching process is typically based on similarity constrains (feature-based or intensity-based). Nevertheless, in epipolar geometry, a point in the right image can be associated with many points in the left image (false matches). This problem is particularly relevant in the measurement of free-form surfaces due the absence of particularities in the scene, used as similarity points [3]. Some calibration procedures can solve the matching problem by intersecting the epipolar line with the laser line in the image [2]. 
This paper presents a non-contact 3D acquisition system based on stereo vision and laser triangulation. The major contribution of the paper is the presentation of the main development stages of an active vision 3D acquisition system. A set of algorithms was developed for each of these stages:

- A calibration procedure for the acquisition system, based on laser line projection plane and stereo vision correspondence oriented towards laser line detection. The calibration procedure, while using only laser-coplanar points, is oriented towards laser line detection, allowing the matching of two images on the laser plane without the use of a rigid motion equation and correcting two kinds of distortion: radial distortion and distortion provoked by the orientation of the camera relative to the laser line projection plane;

- A post-processing module, with an algorithm for data gap filling based on cubic spline interpolation and 3D surface reconstruction based on data hierarchical space decomposition.

The final result of the above contribution was a very accurate $3 \mathrm{D}$ measurement system with a resolution of $0.5 \mathrm{~mm}$.

The paper is divided in the following sections: Sect. 2 describes the developed mechanical prototype, the control system and the configuration of the scanning arm; Sect. 3 explains the calibration procedure developed in two subsections: horizontal and vertical image correction, and real world relationship; Sect. 4 presents an algorithm for laser line detection in surfaces with high laser line absorption; Sect. 5 presents the post-processing module developed, which includes an algorithm for laser line data gap fill and 3D surface reconstruction algorithm. Section 6 shows three practical analyses/tests of the developed acquisition system: the first set checks the system accuracy; the second compares the implemented system with a contact measurement machine (CMM); the third shows the acquisition system results in three different samples of road pavement. Finally, Sect. 7 presents some conclusions about the developed system.

\section{Mechanical prototype and control system}

The mechanical prototype built is a 3 degree freedom machine with a working area of $1,000 \times 1,000 \times 500 \mathrm{~mm}$ (Fig. 1a), controlled by a PC-based motion control system. The machine control system is based on two control loops one for each axis. The function of the first loop is to control of the axis speed, while the second loop controls the axis position. Each axis (corresponding to a toothed belt system) is composed by one servomotor, one resolver coupled to the servomotor, one power driver (SEW-MOVIDRIVE MDX 60B) and two inductive end limit switches. The three axes are connected to the PC through a 4-Axis Servo Controller for the PCI bus (National Instruments PCI 7344).

The scanning arm is composed by two CCD firewire cameras, with a resolution of $640 \times 480$, using $8 \mathrm{~mm}$ focal length lenses and a $635 \mathrm{~nm}$ wavelength, $1 \mathrm{~mW}$ power laser line. The laser is located at the same physical distance from both cameras, and the cameras are oriented at an angle of $30^{\circ}$ to the horizontal line (Fig. 1b).

The machine scanning movements are user-controlled by software developed in Visual C++. The software consists of three main functions: generation of scanning paths (Fig. 1c), data acquisition (Sects. 3, 4) and data post-processing (Sect. 5).

The generation of scanning paths is directly related to the machine control system. First, the user sets the desired scanning movements and sends the selected scanning path and the value of the acquisition rate to the motion control system. The motion control system, after receiving these parameters, moves the axis to start position. When the axis reaches start position, the motion system initiates the scanning movement and triggers the cameras with the selected acquisition rate. The axis position is saved whenever the trigger of the camera is set-associating the acquired line points with the position coordinates ( $x, y$ and $z$ ).

The data acquisition and the data post-processing are explained in the following sections.

\section{Calibration procedure}

Most of the techniques used for camera calibration are normally based on the pinhole camera model. In the first step, the camera image is transformed into an ideal (undistorted) image, using perspective projection with pinhole camera geometry, thus taking into account the intrinsic camera
Fig. 1 Mechanical prototype: a 3D machine; b scanning arm; c user interface for generating scanning paths

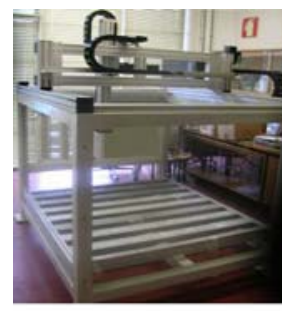

a)

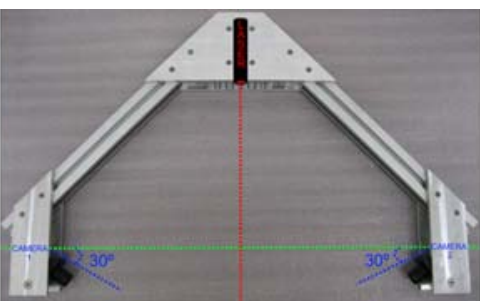

b)

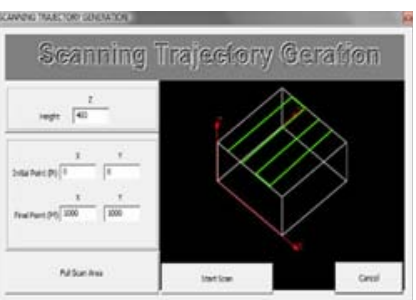

c) 
Fig. 2 Calibration procedure illustration. Representation of a pattern point $(\mathrm{P})$ in both camera images before the calibration procedure (P1-image in top left corner and $\mathrm{P} 2$-image in the top right corner), and representation of the same pattern point $(\mathrm{P})$ in both camera images after the horizontal and vertical steps of the calibration procedure $\left(\mathrm{P}^{\prime}\right.$ - image in the bottom left corner and $\mathrm{P} 2^{\prime}$ - image in the bottom right corner). The system selected vision field is represented in the pattern and the two top images in blue and corresponds to the complete image of both bottom images

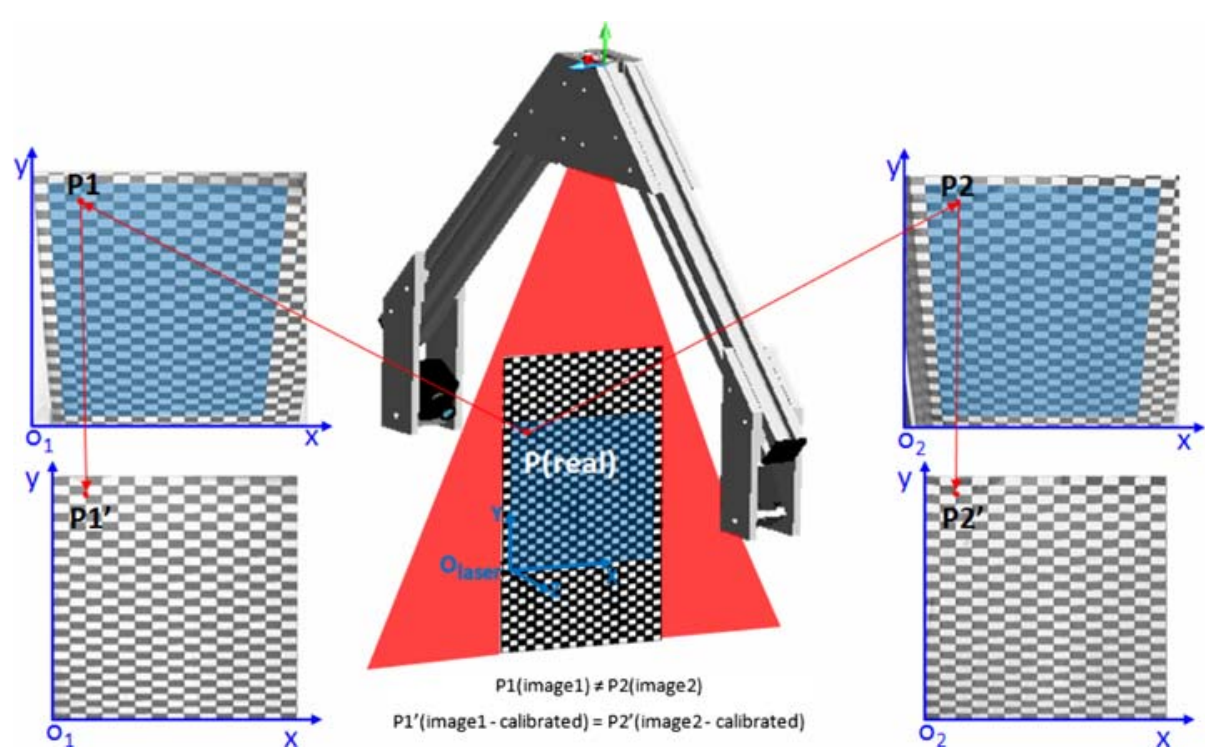

parameters and modelling lens distortion, which can be of two kinds: radial and tangential. Then, the calibration parameters $\mathrm{R}$ (rotation matrix) and $\mathrm{T}$ (translation vector) are defined, allowing, through a rigid equation, the transformation of the image world coordinate system in the object world coordinate system.

In the proposed calibration procedure, only radial distortion will be considered because it is the most relevant distortion to industrial machine vision applications, and only the laser line projection plane is calibrated because the measurements are only made along the plane defined by the laser line. The laser is located at the same physical distance from both cameras, and the cameras are oriented at $30^{\circ}$ to the horizontal line (Fig. 2). Although, the system is mechanically adjusted (distance between cameras and laser, angles between cameras and laser line), the acquired images of both cameras showed that, in the laser line projection plane, the system was far from being calibrated, presenting the following problems:

- A point $P($ real $)$ in the $O_{\text {laser }}$ (laser coordinated system), corresponds to a point $P 1$ in the $O_{1}$ (camera 1 coordinated system), and another point $P 2$ in the $O_{2}$ (camera 2 coordinated system), $P 1$ being different from $P 2$;

- Perfectly visible radial distortion in some parts of the image, adding to the previous problem;

- Formation of image dead zones-problem caused by the relative angle of the cameras to the laser plane. This distortion means that the horizontal length of the vision field at the top of the image is smaller than the horizontal length of the vision field at the bottom of the image. This problem only matters because the system to be calibrated is assembled in a 3D freedom machine that will carry out readings on parallel surface planes.
To overcome these problems, a calibration procedure was developed which calibrates each image, asserting that a point in $\mathrm{O}_{1}\left(\mathrm{P}^{\prime}\right)$ is equal to a point in $\mathrm{O}_{2}\left(\mathrm{P}^{\prime}\right)$ on the laser plane, eliminating the radial distortion caused by the lenses and image dead zones.

For calibration, a pattern was created with $10 \times 5 \mathrm{~mm}$ black and white rectangles. The pattern was printed on a transparency sheet and placed between two sheets of glass with a thickness of $1.2 \mathrm{~mm}$, all the rectangles being co-planar and visible to both the cameras. The pattern must then be aligned along the laser line projection plane allowing an easy match between the same image points seen by both cameras, which is the basis of this calibration procedure (Fig. 3).

The calibration procedure starts with the capture of a pattern image by each camera. After both images have been acquired, a valid vision field is defined for each camera (Fig. 3). The selected vision field must be the same for both cameras, the horizontal and vertical limits of the selected vision field must correspond to the same physical points of the pattern.

The third step of the calibration procedure consists in the horizontal calibration of the acquired images. In this step, the horizontal correction of the images is made (Fig. 4a), resulting in two new horizontally undistorted images (Fig. 4b).

The fourth step concerns the vertical calibration of the resultant images of the previous step. These two new images are horizontally and vertically distortion-free (Fig. 4c).

At the end of above four steps, pixels with the same coordinates in images acquired from each camera correspond to the same physical location in the pattern. As a result, data obtained from an image acquired by camera 1 can now be used to complement data extracted from the corresponding image acquired by camera 2 , particularly in the case of object occlusion problem. 
Fig. 3 Calibration set up: scanning arm and pattern assembling. The vision field is represented in blue and the laser plane in red

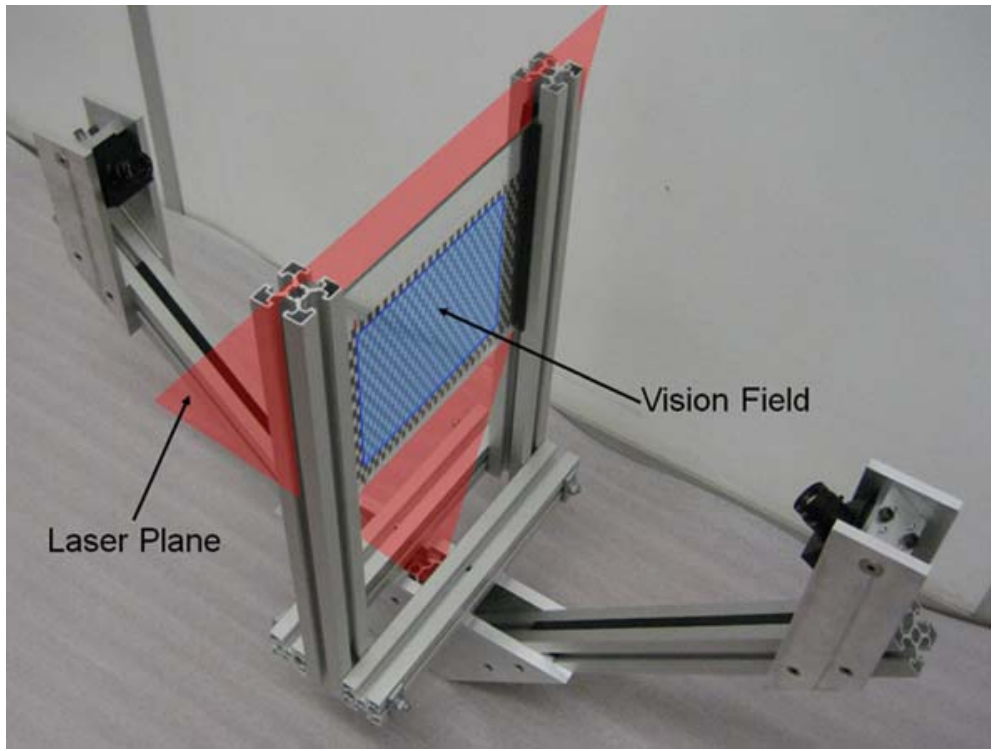

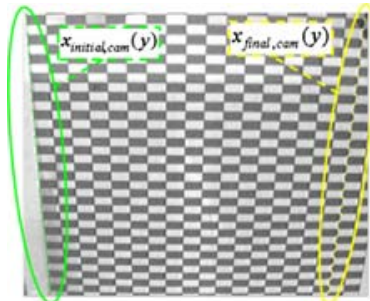

a)

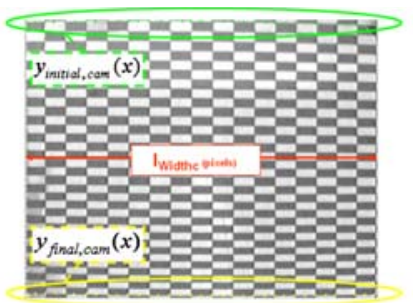

b)

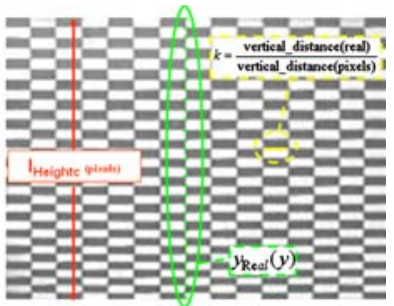

c)
Fig. 4 Calibration images of one camera: a horizontal image correction (green initial horizontal limits of image, yellow horizontal limits of image); b vertical image correction (green initial vertical limits of image, yellow vertical limits of image, red corrected image width); c Real world coordinates correspondence (green correspondence between corrected image points and pattern points, yellow $k$ constant determination, red corrected image height)
In the last step of the calibration procedure, it is necessary to set a relationship between the image points and the pattern points (Fig. 4c) - a linear relationship in the horizontal component and a polynomial relationship in the vertical component. The determination of those relationships needs to be applied to one image only.

The horizontal image correction, vertical image correction and real world relationships are described in detail in the following sections. The calibration procedure also includes the automatic laser line detection as will be described in Sect. 4 .

\subsection{Horizontal and vertical image correction}

The horizontal and vertical image correction steps for each camera (Fig. 4a, b) are executed sequentially, using the same algorithm which comprises the following steps:

- Acquisition of a set of points from the image (green points in Fig $4 \mathrm{a}$-horizontal image correction, green points in
Fig $4 \mathrm{~b}$ - vertical image correction), which will be zeros in the image after correction;

- Polynomial determination that translates the relation between the point's horizontal and vertical positions according to Eq. 1;

- Acquisition of a set of points from the image (yellow points in Fig 4a-horizontal image correction, yellow points in Fig $4 \mathrm{~b}$ - vertical image correction), which will be the end limits of the image after correction;

- Polynomial determination that translates the relation between the point's horizontal and vertical positions according to Eq. 2;

- Generation of a new corrected image, with horizontal and vertical length given by (3) (Fig. 4b-horizontally corrected image; Fig. 4c-vertically corrected image), and new image points position given by (4) ( $y$ position remains equal - horizontal image correction; $x$ position remains equal—vertical image correction). 


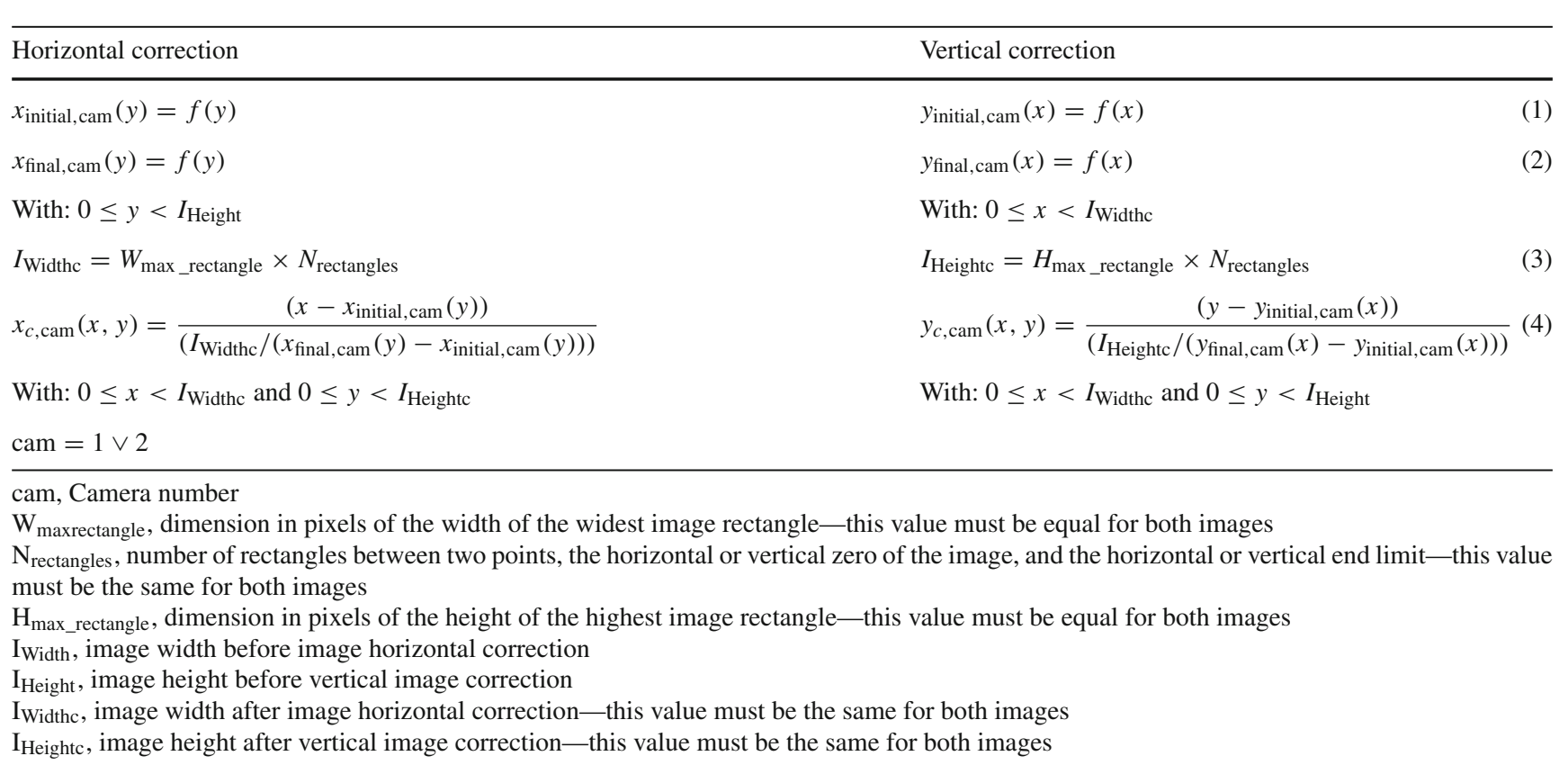

\subsection{Real world relationship}

The relationship between the corrected image points and the points of $O_{\text {laser }}$, in $x$ coordinate, is obtained by (5), where $k$ is a constant. This constant, $k$, is determined by the division of the vertical length of one rectangle of the pattern of the calibrated image and the vertical length of one rectangle of the physical pattern (10 $\mathrm{mm}$ in the pattern used).

$x_{\text {Real }}=k \times x, \quad 0 \leq x<I_{\text {Widthc }}$

In $y$ coordinate, the relationship is obtained by (6), where $f(y)$ is a polynomial. This polynomial is defined by the acquisition of a set of vertical points in the corrected image. These points must represent a point on each horizontal edge of the rectangle in the corrected image, once the real distance between the edges is known (through the physical pattern).

$y_{\text {Real }}(y)=f(y), \quad 0 \leq y<I_{\text {Heightc }}$

The selection of points in each step of the calibration procedure was done manually. Several methods for point setting were tested. The best method, resulting in a smaller mean squared error, was a 3 degree polynomial approximation (7). For the determination of the polynomial, in both calibration steps, the least-squares method was used.

$y=b+c_{1} x+c_{2} x^{2}+c_{3} x^{3}$

$b$ and $c_{1} \ldots c_{3}$ are constants

\section{Laser line detection}

The accuracy of a 3D measurement, which uses laser scanners, is significantly affected by the detection of the laser line.
The energy pattern of such a line corresponds to a Gaussian profile. The laser line detection method presented here is executed in two steps.

The first step, applied to both images, is carried out before calibration process. This step is characterized by the first vertical derivative image calculation. Therefore, each image column is derived and the laser is detected (Fig. 5), using three validating conditions:

- To search in the derived signal (column) for positive to negative transitions and the associated peaks;

- To verify if the distance between peaks is greater than $\Delta_{\text {Laser_Height }}$-maximum number of vertical laser pixels;

- To calculate the amplitude between peaks and to verify if the amplitude is greater than $\Delta_{\text {Laser_Intensity }}$-laser minimum intensity relative to the surface on which it occurs.

If the three conditions are positive, the vertical pixels of the analysed column, between transitions, are placed in white and

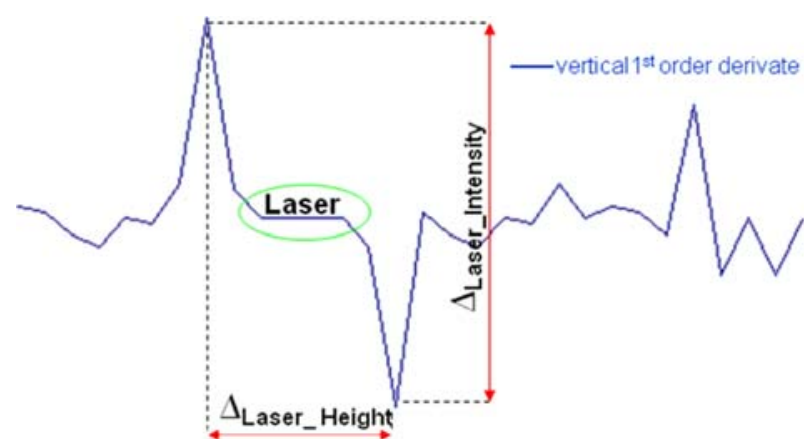

Fig. 5 Representation of image vertical first-order derivative and laser line detection 


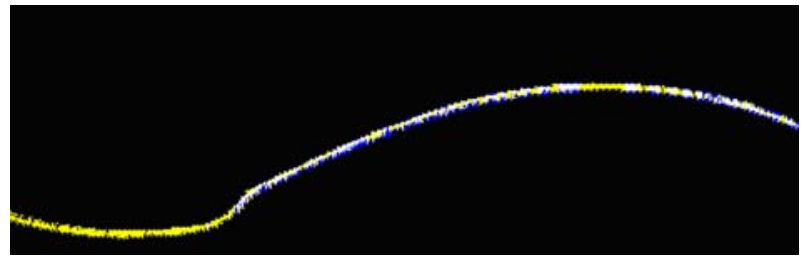

Fig. 6 Laser line detection result, by both cameras, after execution of the horizontal and vertical image calibration and logical OR: yellow camera 1 image, blue camera 2 image, white camera 1 image and camera 2 image intersection

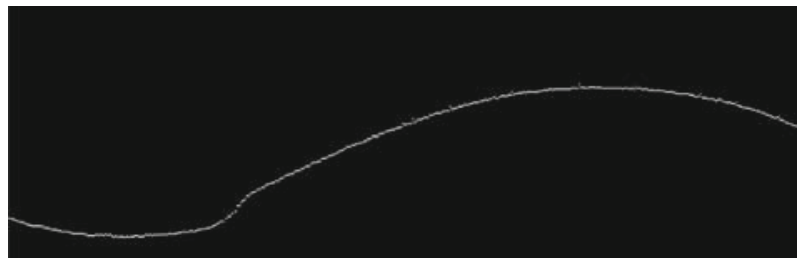

Fig. 7 Image resulting from the logic operation

the remaining in black. In the event of detecting more than one zone with valid parameters in the same column, the one with greater $\Delta_{\text {Laser_Intensity }}$ is chosen. The value of the parameter $\Delta_{\text {Laser_Height }}$ is attributed according to the vertical resolution of the camera, the laser power and the distance between camera and laser. The value of the parameter $\Delta_{\text {Laser_Intensity }}$ is defined by analysing the intensity of the laser line over the material on which the measurements will be taken. After the laser line detection in both images, Sect. 3.1 of the calibration procedure is executed. Then, a logic OR operation is carried out between the two calibrated images (Fig. 6).

The second step of the laser line detection consists in the detection of the laser line centre, which corresponds to the average value between the maximum and the minimum values. This step is carried out after Sect. 3.1 and before Sect. 3.2 of the calibration procedure. Then, the laser line centre is detected from the resultant image of the logical operation (Fig. 7). This image is then ready for execution in Sect. 3.2, the final step of the calibration procedure.

\section{Post-processing module}

The post-processing module is based on the execution of two algorithms for high accuracy 3D road surface reconstruction. The first algorithm is responsible for filling the information gaps in the acquired laser line; the second reconstructs the 3D surface.

The objects used in the practical tests were samples of road pavements, which present a complex geometry, hiding the laser line of the cameras in some narrow areas (occlusion problem), originating small information gaps in the acquired laser line. The correction of the information gaps, finding the missing values, is achieved by using a cubic spline interpolation algorithm.

The interpolation is based on the previous and subsequent gap information of the acquired laser line. As a result of the application of this algorithm, the data processed do not present any gaps and are ready for surface reconstruction. For this purpose, another algorithm based on 3D data space organization was developed, allowing the generation of square and triangular surface meshes with different resolutions.

\subsection{Data gap filling}

The cubic spline interpolation method consists in dividing the interest interval (previous and subsequent gap information) in several sub-intervals and do a curve fitting using a polynomial of degree 3 [7]. In this case, for information gap filling, intervals with 12 sample points were defined, 6 points before and 6 points after the information gap. Assuming that the points are $\left(\left(x_{0}, f\left(x_{0}\right)\right), \ldots,\left(x_{11}, f\left(x_{11}\right)\right)\right)$ and $f^{\prime \prime}\left(x_{0}\right)=f^{\prime \prime}\left(x_{11}\right)=0$, the second derivatives for the remaining points can be calculated through (8). The cubic function for the calculation of information gap points is defined by (9) (for $i$ equal to 6), where $f_{6}(x)$ values are obtained from $x$ values equally spaced.

$$
\begin{aligned}
& \left(x_{i}-x_{i-1}\right) f^{\prime \prime}\left(x_{i-1}\right)+2\left(x_{i+1}-x_{i-1}\right) f^{\prime \prime}\left(x_{i}\right) \\
& \quad+\left(x_{i+1}-x_{i}\right) f^{\prime \prime}\left(x_{i+1}\right) \\
& =\frac{6}{\left(x_{i+1}-x_{i}\right)}\left[f\left(x_{i+1}\right)-f\left(x_{i}\right)\right]+\frac{6}{\left(x_{i}-x_{i-1}\right)} \\
& \quad \times\left[f\left(x_{i-1}\right)-f\left(x_{i}\right)\right] \quad \text { with } i=1,2,3, \ldots, n-1 \quad(8) \\
& f_{i}(x)=\frac{f^{\prime \prime}\left(x_{i-1}\right)}{6\left(x_{i}-x_{i-1}\right)}\left(x_{i}-x\right)^{3} \\
& \quad+\frac{f^{\prime \prime}\left(x_{i}\right)}{6\left(x_{i}-x_{i-1}\right)}\left(x-x_{i-1}\right)^{3} \\
& \quad+\left[\frac{f\left(x_{i-1}\right)}{x_{i}-x_{i-1}}-\frac{f^{\prime \prime}\left(x_{i-1}\right)\left(x_{i}-x_{i-1}\right)}{6}\right](x i-x) \\
& \quad+\left[\frac{f\left(x_{i}\right)}{x_{i}-x_{i-1}}-\frac{f^{\prime \prime}\left(x_{i}\right)\left(x_{i}-x_{i-1}\right)}{6}\right]\left(x-x_{i-1}\right)
\end{aligned}
$$

\subsection{D surface reconstruction}

The acquisition system presented captures a 3D data set of unorganized points. Given that the surface acquisition is over one plane, the proposed reconstruction method transforms a $3 \mathrm{D}$ problem into a $2 \mathrm{D}$ problem. The developed method is based on the algorithms proposed by $[8,9]$, but with a difference in the data set organization. Here, it is proposed a quadtree (Fig. 8) replacing the octree proposed by [9]. This difference significantly reduces the computational time because all operations are done on 2D. 
Fig. 8 Image of the surface reconstruction process: b connectivity graph and c surface a Quadtree data structure,

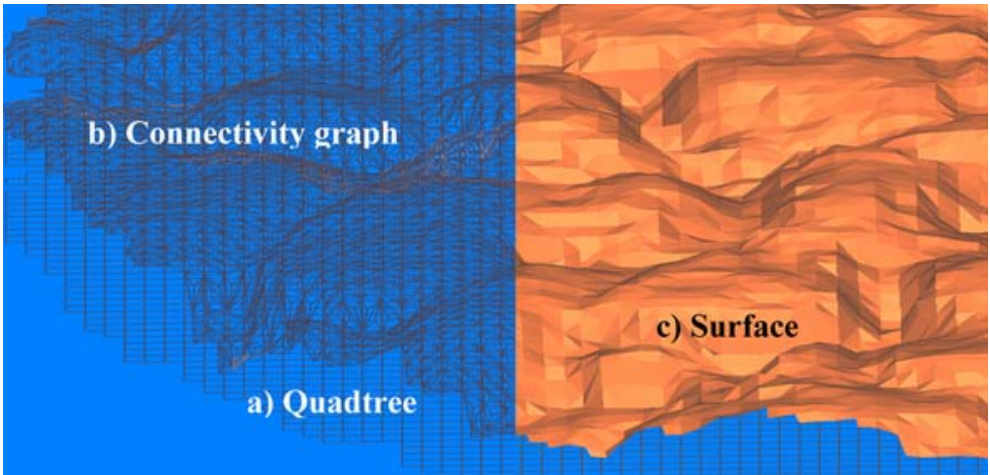

The 3D surface reconstruction method steps are: (1) create a 2D grid; (2) produce a connectivity graph based on a search for four neighbouring squares, each four square; (3) surface reconstruction.

In the first step, the acquired points are aggregated in one square. Square dimensions are set by the maximum value of the major distance between the axes of the scanned surface (belonging to the scanned plane). The square is repeatedly divided into smaller squares, until the stop criterion is reached (based on the user defined resolution), thus creating an uniform structure with a specific resolution. After the quadtree creation, the centroid of each square is calculated, based in the geometrically distribution of the points. The next step consists in searching for four neighbouring squares for each square. The goal of this search is to associate each square's centroid point with a doubly connected edge list (DCEL). Finally, each edge (belonging to DCEL) is associated with a surface, using the minimal loop strategy [9].

To improve the surface appearance, a triangular subdivision on each created surface is performed. Each vertex of the triangle vertices has a normal vector associated. The vector is obtained by a normalized mean of the normal vectors associated with the triangles where the edge belongs.

\section{Results and discussion}

To demonstrate the effectiveness of the developed acquisition system, two different measurement analyses were made and three different types of road pavement were acquired and reconstructed.

The first analysis carried out by the developed system had, as main incidence, the extremities of the system's vision field. As was previously verified, it was at the extremities of the images that the radial distortion caused by lens and the distortion caused by the cameras orientation to the laser line projection plane were more perceptible. Therefore, two calibrated objects, with different types of geometry and dimensions, were used for test measurements in this system.

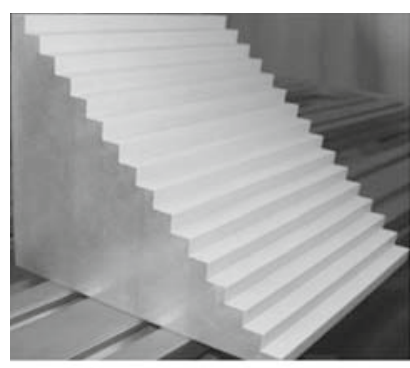

a)

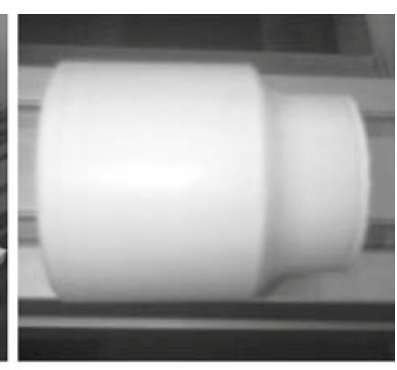

b)
Fig. 9 Calibres used in the calibration procedure: a Stepper calibre, b cylindrical with variable radius calibre

The selected objects present the following geometric and dimensional characteristics:

- Calibre 1-Stepper calibre (Fig. 9a)—with horizontal and vertical steps of approximately $10 \mathrm{~mm}$;

- Calibre 2-Variable radius cylinder calibre (Fig. 9b) with two control radius points.

A high precision contact measure machine (CMM) was used to obtain the height and length values of each step of calibre 1 and the two radius values of calibre 2 (Mitutoyo, Class 0, Precision $0.1 \mu \mathrm{m}$ ).

A set of measurements of calibre 1 (height and length) was achieved using the developed system. The measurements were made with the calibre placed in different positions of the image, with special incidence on the image borders, because those are the regions of greatest distortion. In Fig. 10a, b, the measurements are shown along $X$ axis (horizontal) and $Y$ axis (vertical), respectively. The statistical results are presented in Table 1.

To obtain the value of the radius of the circles of calibre 2 , three measurements were made in different points of each circle. The measurements of calibre 2 were made in different positions of the image, with special incidence on the image extremities. Figure 10c, d shows the measurement results 
Fig. 10 Calibration results: a Chart with five calibre1 horizontal step measurements ( $X$ axis) on five distinguished image points (M1 to M5), b chart with five calibre1 vertical step measurements $(Y$ axis) on five determined image points (M1 to M5), c and $\mathbf{d}$ Chart with radius measures in five image points, using calibre 2

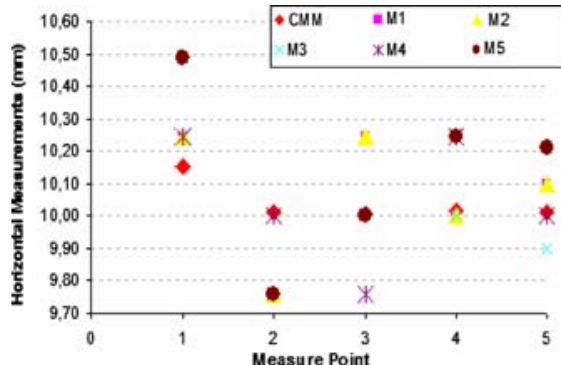

a)

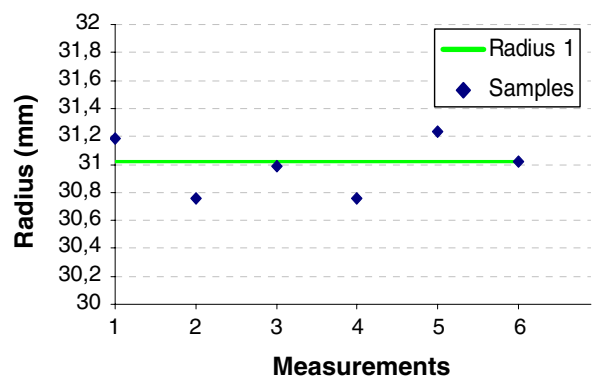

c)

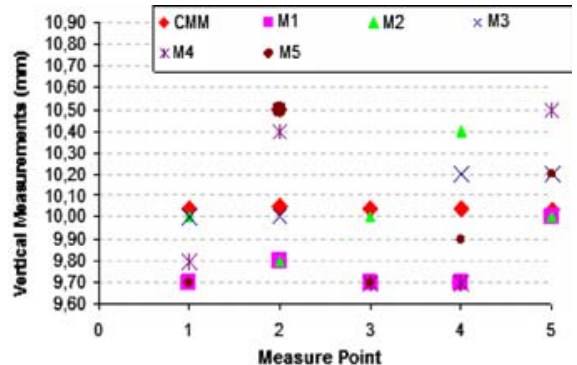

b)

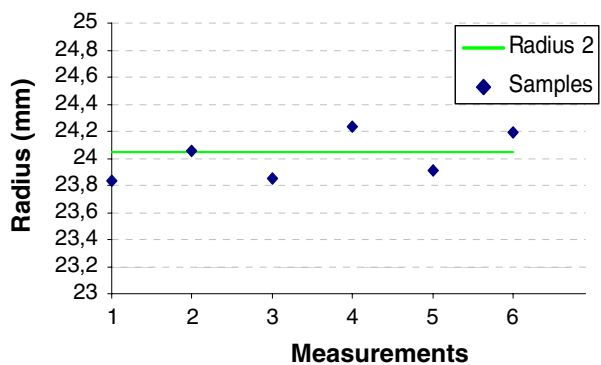

d)
Table 1 Calibre 1: horizontal and vertical measurement error results

\begin{tabular}{lrlll}
\hline Error measure type & Mean & Min & Max & SD \\
\hline Horizontal (mm) & 0.05 & -0.25 & 0.34 & 0.19 \\
Vertical (mm) & -0.03 & -0.34 & 0.36 & 0.23 \\
\hline
\end{tabular}

Table 2 Calibre 2: radius measurement error results

\begin{tabular}{lllll}
\hline Calibre radius & Mean & Min & Max & SD \\
\hline $1(\mathrm{~mm})$ & 0.03 & -0.22 & 0.27 & 0.21 \\
$2(\mathrm{~mm})$ & 0.04 & -0.18 & 0.21 & 0.17 \\
\hline
\end{tabular}

made by the system for, respectively, radius 1 and 2 . The statistical results are shown in Table 2.

From the results analysis, it is possible to say that

- The system presents a $0.25 \mathrm{~mm} /$ pixel horizontal resolution and a $0.33 \mathrm{~mm} /$ pixel vertical resolution, in a vision field of $170 \times 160 \mathrm{~mm}$.

- The maximum error is smaller than 2 pixels, which expresses a system reading error smaller than $0.5 \mathrm{~mm}$, in both orientations (vertical and horizontal). The system error is subject to improvement by using higher resolution cameras or lenses that reduce the vision field. Therefore, increasing the system resolution results in a less significant pixel error.

- The standard deviation is approximately the same in both calibre measurements.

- The mean error is practically zero in both measurements, suggesting that the error fluctuates above and below the real value.

- The mean error is smaller than the system resolution.
- Easy laser line detection on surfaces with high level of laser line absorption and complex geometry.

The second analysis of the system developed was executed on a road surface texture. This analysis is intended to demonstrate the possibility of a practical application of this system in the dimensional inspection of this type of surface. For this purpose a comparative study of a complex line profile was made using a CMM (also used for calibre measurement) and the developed system (Fig. 11). The sample distance was of $1 \mathrm{~mm}$. The acquired sample presents a complex profile. This reality frustrates the profile acquisition by the CMM in some narrow concavities, as is the case of the intervals $\{23,28\}$ and $\{30,40\}$.

Tables 3 and 4 show the statistical comparison between the two acquisition systems. Looking at the results it is possible to emphasize that:

- The mean value of the CMM system is $0.36 \mathrm{~mm}$ greater than the mean value of the developed system. This fact could be influenced by the scanner system error and the CMM difficulty in accessing narrow concavities.

- The maximal and minimum vertical values measured in several acquisition points present similar results in both systems.

- The maximal acquisition point has almost the same value for both systems.

- The standard derivation is higher in the system developed than in the CMM system; this is due to the same factors that affect the measured mean value.

Figure 12 shows the acquisition and reconstruction results of three different types of road pavement. The height of each 
Fig. 11 This graph establishes a comparison between a line points acquisition carried out by the developed scanner system and the CMM over road profile surface

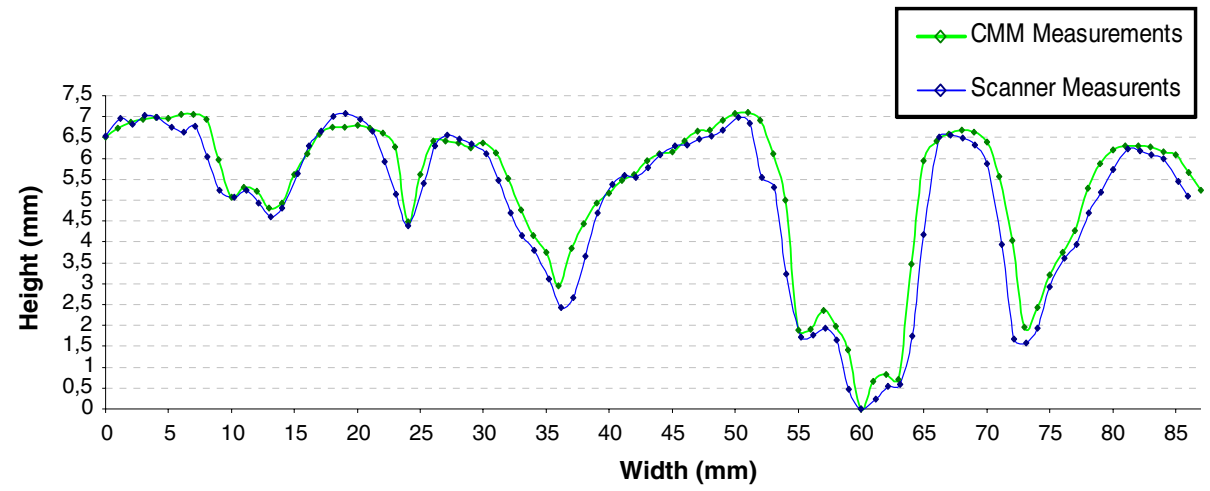

Table 3 Comparison between points acquired by the CMM and by the system developed

\begin{tabular}{lllll}
\hline Measurement system & Mean & Min & Max & SD \\
\hline CMM (mm) & 5.29 & 0.00 & 7.09 & 1.76 \\
Developed system (mm) & 4.93 & 0.01 & 7.08 & 1.92 \\
\hline
\end{tabular}

Table 4 Errors in distances between point acquisition by the CMM and the system developed

\begin{tabular}{lllll}
\hline Measurement & Mean & Min & Max & SD \\
\hline Error $(\mathrm{mm})$ & -0.13 & 0.24 & 0.05 & 0.07
\end{tabular}

sample is represented by a colour grid. The mesh resolution selected for these samples was $0.5 \times 0.5 \mathrm{~mm}$ and the mesh type was triangular.

\section{Conclusion}

This paper presents a data acquisition system based on stereo vision and laser triangulation. The proposed system is composed of two CCD firewire cameras, with a resolution of $640 \times 480$, using $8 \mathrm{~mm}$ focal length lenses and a $635 \mathrm{~nm}$ wavelength, $1 \mathrm{~mW}$ power laser line, presenting a reading error smaller than $0.5 \mathrm{~mm}$. This system includes also different
Fig. 12 Acquisition and reconstruction results of three different road pavements by the developed acquisition system: a "Porous Asphalt" picture, b "Porous Asphalt" surface reconstruction, c "Semi-Porous Asphalt" picture, d "Semi-Porous Asphalt" surface reconstruction, $\mathbf{e}$ "Dense Asphalt" picture, f "Dense Asphalt" surface reconstruction

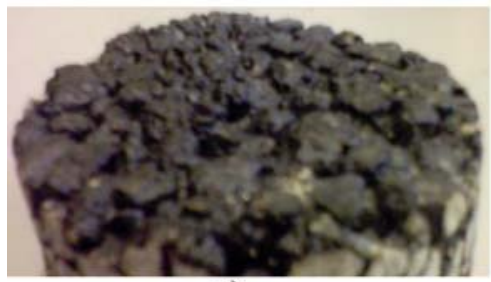

a)

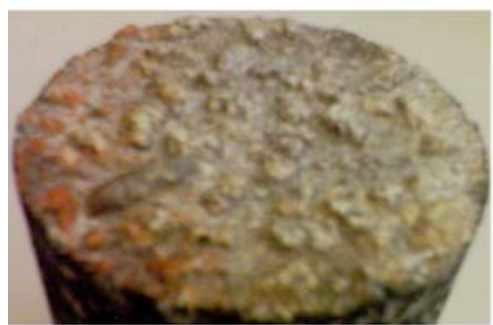

c)

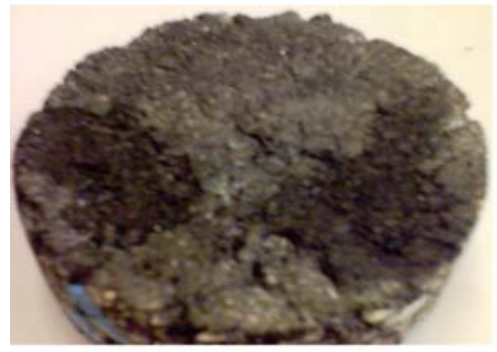

e)

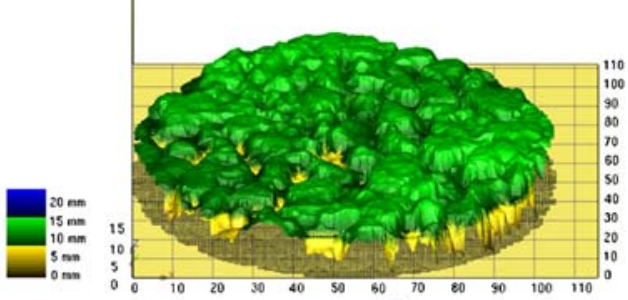

b)

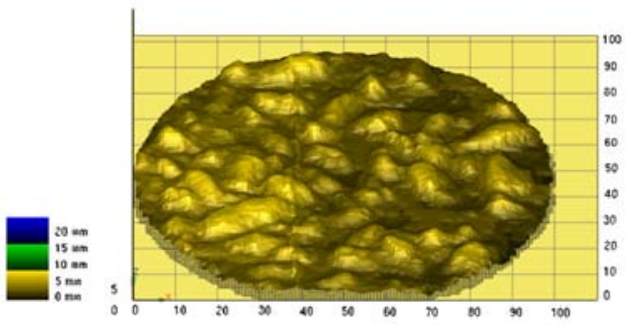

d)

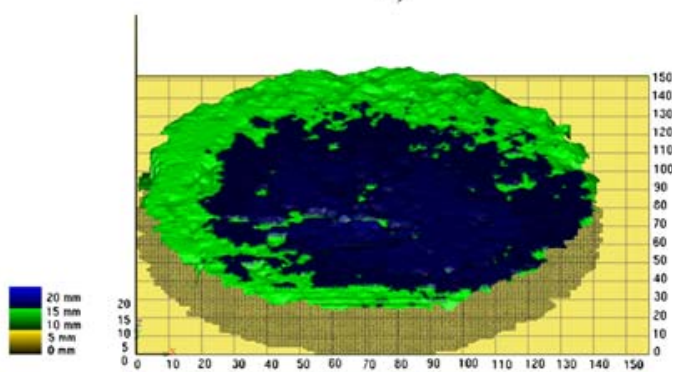

f) 
software modules allowing the acquisition and 3D reconstruction of objects with high level of laser line absorption and complex geometry.

The calibration procedure, while using only laser-coplanar points, is oriented towards laser line detection, allowing the matching of two images on the laser plane without the use of a rigid motion equation and correcting two kinds of distortion: radial distortion and distortion caused by the orientation of the camera relative to the laser line projection plane.

The proposed post-processing module presents two new algorithms. The first is used to fill the gaps in the acquired laser line decreasing/eliminating the occlusion problem typical of surfaces with narrow concavities. The second is used for 3D surface reconstruction, producing triangular or quadrangular meshes (used for finite element analysis) with different resolutions.

The acquisition characteristics of the developed system associated with the algorithms presented for treatment and reconstruction of surfaces allow its usage for road surface textures acquisition, for macro and mega textures analysis, as was demonstrated by results presented in Sect. 6 .

Acknowledgments This work has been supported by "Fundação para a Ciência e a Tecnologia" (Portugal) through a Ph.D. grant, reference SFRH/BD/18155/2004.

\section{References}

1. Aguilar, J., Torres, F., Lopes, A.: Stereo Vision for 3D measurement: accuracy analysis, calibration and industrial applications. Measurement 18(4), 193-200 (1996)
2. Barone, S., Bruno, A.: Stereo Vision and laser stripers for threedimensional surface measurements. XVI Congreso International de Ingeniería Gráfica (2004)

3. Devernay, F., Faugeras, O.: Computing differential properties of 3-D shapes from stereoscopic images without 3-D models. In: CVPR'94, pp. 208-213 (1994)

4. França, J., Gazziro, M., Ide, A., Saito, J.: A 3D Scanning System Based on laser Triangulation and Variable Field of View. IEEE Conference on Image Processing (2005)

5. Heikkila, J., Silve, O.: A four-step camera calibration procedure with implicit image correction. In: Proceedings IEEE CVPR'97, pp. 1106-1112 (1997)

6. Lenz, R., Tsay, R.: Techniques for calibration of scale factor and image center for high accuracy 3-D machine vision metrology. IEEE Trans. Pattern Anal. Mach. Intell. 10(5) (1988)

7. http://online.redwoods.cc.ca.us/instruct/darnold/laproj/Fall98/ SkyMeg/Proj.PDF

8. Hugues, H.: Surface reconstruction from unorganized points. University of Washington, Washington (1995)

9. Azernikov, S., Miropolsk, A., Fischer, A.: Surface reconstruction of freeform objects based on multiresolution volumetric method. In: ACM Symposium on Solid and Physical Modeling, pp. 115-126 (2003)

10. Sturm, P., Maybank, S.: On plane-based camera calibration: a general algorithm, singularities, applications. In: Proceedings IEEE CVPR'99, pp. 432-437 (1999)

11. Tsai, R.: A Versatile camera calibration technique for high accuracy 3D machine vision metrology using off-the-shelf TV cameras and lenses, IBM Res. Rep. RC 51342 (1985)

12. Hong-Tzong, Y., Chun-Yan, C., Wilhelm, R.: Registration and integration of multiple laser scanned data for reverse engineering of complex 3D models. Int. J. Prod. Res. 38(2), 269-285 (2000)

13. Zhang, Z.: A flexible new technique for camera calibration. IEEE Trans. Pattern Anal. Mach. Intell. 22(11), 1330-1334 (2000) 